\title{
Prenatal Exposure to High Cortisol Induces ADHD-like Behaviors with Delay in Spatial Cognitive Functions during the Post-weaning Period in Rats
}

\author{
Sang-Chan Jeon ${ }^{1 \dagger}$, Hye-Ji Kim ${ }^{1 \dagger}$, Eun-A Ko ${ }^{1}$ and Sung-Cherl Jung ${ }^{1,2,3 *}$ \\ ${ }^{1}$ Department of Physiology, School of Medicine, Jeju National University, ${ }^{2}$ Interdisciplinary Graduate Program in \\ Advanced Convergence Technology \& Science, Jeju National University, ${ }^{3}$ Institute of Medical Science, \\ Jeju National University, Jeju 63243, Korea
}

\begin{abstract}
High levels of cortisol in blood are frequently observed in patients with major depressive disorders and increased cortisol level induces depressivelike symptoms in animal models. However, it is still unclear whether maternal cortisol level during pregnancy is a critical factor resulting in neuropsychiatric disorders in offspring. In this study, we increased cortisol level in rats by repetitively injecting corticosterone subcutaneously (Corti. Mom, $20 \mathrm{mg} / \mathrm{kg} /$ day) during pregnancy and evaluated the behavioral patterns of their pups (Corti.Pups) via forced swimming (FS), open field (OF), elevated plus maze (EPM) and Morris water maze (MWM) tests during the immediate post-weaning period (postnatal day 21 to 25). In results, corticosterone significantly increased plasma cortisol levels in both Corti.Moms and Corti.Pups. Unlike depressive animal models, Corti.Pups showed higher hyperactive behaviors in the FS and OF tests than normal pups (Nor.Pups) born from rats (Nor.Moms) treated with saline. Furthermore, Corti.Pups spent more time and traveled longer distance in the open arms of EPM test, exhibiting higher extremity. These patterns were consistent with behavioral symptoms observed in animal models of attention deficit hyperactivity disorder (ADHD), which is characterized by hyperactivity, impulsivity, and inattention. Additionally, Corti.Pups swam longer and farther to escape in MWM test, showing cognitive declines associated with attention deficit. Our findings provide evidence that maternal cortisol level during pregnancy may affect the neuroendocrine regulation and the brain development of offspring, resulting in heterogeneous developmental brain disorders such as ADHD.
\end{abstract}

Key words: Cortisol, Maternal depression, ADHD, Hyperactivity, Impulsivity, Cognitive impairment

\section{INTRODUCTION}

Over the years, it has been reported that maternal stress during pregnancy affects the physical development and psychiatric health of prenatal and postnatal infants. In human studies, post-traumatic stress of pregnant mothers was associated with cortisol accumulation in infant hair and dysregulation of maternal hypothalamic-

Submitted December 1, 2020, Revised January 28, 2021,

Accepted February 11,2021

* To whom correspondence should be addressed.

TEL: 82-64-754-3834, FAX: 82-64-702-2687

e-mail:jungsc@jejunu.ac.kr

These authors contributed equally to this article. pituitary-adrenal (HPA) axis affects maternal health during pregnancy and fetal development $[1,2]$. A recent study in children aged 6 to 7 years reported that higher cortisol levels in the hair were specifically correlated with a lower volume of CA3 and dentate gyrus and associated with cumulative glucocorticoid levels [3]. Exposure to excessive glucocorticoids during pregnancy directly induces the dysregulation of placental lipid transport, metabolism and glucose uptake and causes structural and functional abnormalities during fetal development [4]. These studies suggest that developmental disabilities in children with neuropsychiatric disorders are correlated with maternal stress during pregnancy.

Higher level of plasma cortisol is a well-known biological indicator of depressive disorders in human and animal models. Particularly, maternal depression induces dysregulation of HPA axis
Copyright $(\odot)$ Experimental Neurobiology 2021. www.enjournal.org
This is an Open Access article distributed under the terms of the Creative Commons Attribution Non-Commercial License (http://creativecommons.org/licenses/by-nc/4.0) which permits unrestricted non-commercial use, distribution, and reproduction in any medium, provided the original work is properly cited. 
by changing the cortisol responses and affecting the maternal and infant neuroendocrine systems [5-8]. In animal models, stressinduced increase in plasma cortisol was reflected in altered fetal cortisol levels and repetitive injections of corticosterone directly increased the level of blood cortisol, inducing various depressive symptoms $[9,10]$. This cortisol response was dependent on the expression of glucocorticoid receptors. Furthermore, the biological correlation in cortisol response between a mother and offspring seems to be more evident in animal models. A reduced neonatal survival rate and decreased body weight of offspring were reported in depressive female mice with normal fertility and the offspring exhibited impaired neonatal reflexes, memory decline, and anxiety-like emotionality during adulthood [11-14]. These previous reports suggested that abnormalities in the offspring of depressive rodents might be correlated with overexpression of glucocorticoid receptors in the brain, demonstrating that prenatal depression induced long-term detrimental effects on behavioral performance and brain development. Recently, it was reported that prenatal stress increases anxiety, depression, and stress-related hyperactive behaviors as well as deficits in learning and memory function [15-18]. Particularly, spontaneously hypertensive rats (SHRs), which are frequently used in studies of attention deficit hyperactivity disorder (ADHD), showed higher serum levels of progesterone, corticosterone and cortisol than normal rats, suggesting that the endocrine-dependent regulation in developmental nervous system plays a major role in determining behavioral and emotional disorders [19]. However, although neuroendocrine dysregulation has been targeted in studies of psychiatric disorders, it is still necessary to investigate whether maternal stressors during pregnancy directly mediates behavioral and emotional abnormalities in postpartum offspring.

In this study, we purposed to characterize the behavioral phenotypes of rat pups that had prenatally been exposed to high cortisol due to the dysregulation of maternal cortisol response during pregnancy. For this, we tried to induce maternal neuroendocrine dysregulation in rats by repetitively injecting corticosterone during pregnancy and then investigated if the high levels of maternal cortisol might be reflected in postnatal rat pups. Also, according to changed cortisol levels, we observed emotional and behavioral abnormalities associated with psychiatric disorders such as depression or autism in postnatal rat pups. Experimental results were obtained from rats (Corti.Moms) daily injected with corticosterone during pregnancy and their pups (Corti.Pups), and compared with normal rats (Nor.Moms) injected with saline and normal pups (Nor.Pups) born from them. Behavioral evaluations of rat pups were performed via forced swimming (FS), open field (OF), elevated plus maze (EPM) and Morris-water maze (MWM) tests during the immediate post-weaning period (postnatal day 21 to 25$)$.

Based on results, this study clearly suggests the potency of methodological approaches to change maternal cortisol level in studies of neuropsychiatric and developmental brain disorders resulted from neuroendocrine dysregulation.

\section{MATERIALS AND METHODS}

\section{Animals and drug administration}

Female Sprague-Dawley (SD) rats were bred in the animal facility of Medical School, Jeju National University. The environment of the breeding room was automatically controlled at a temperature of $23 \pm 3^{\circ} \mathrm{C}$ and $50 \pm 10 \%$ humidity under a 12 -h light/dark cycle. Access to food and water was provided ad libitum. One female and one male were mated for breeding. As previously described by lijima et al. in 2010 [9], the Corti.Mom model in this study was developed by the method to induce cortisol-mediated depressive symptoms in adult rodents. Corticosterone $(20 \mathrm{mg} / \mathrm{kg})$ was subcutaneously injected dorsally into 8 9-week-old female rats once a day during pregnancy immediately after confirming fertilization (Fig. 1A). Pups born from Corti.Moms were defined as Corti. Pups. For comparison, pregnant rats that were treated daily with saline $(0.9 \% \mathrm{NaCl})$ instead of corticosterone represented the normal group (Nor.Mom) and their pups were defined as Nor.Pups. Corticosterone (Sigma-Aldrich) that was suspended in $0.9 \% \mathrm{NaCl}$ supplemented with $0.1 \%$ DMSO and $0.1 \%$ Tween 80 , was used for injection. All pups were cared by their mothers for 3 weeks after birth and then used in behavioral tests during the postnatal week 4. All animal experiments and procedures were performed with the approval from the Animal Care and Use Committee of Jeju National University.

\section{Behavioral experiments}

Behavioral experiments involving male and female pups were performed from postnatal day 21 to day 25 (to day 23 in some experiments). Behavioral tests were conducted between 1:30 PM and 4:00 PM and all rat pups were transferred to the testing room 30 min before the trial to habituate to the condition at $22 \sim 24^{\circ} \mathrm{C}$ of air temperature. The test room was soundproof, and the light intensity was set at 200 250 lux with overhead LED fluorescent lamps (20 watt). Before all experiments, rat pups were confirmed to be moving freely in the cage and the order of trials was counterbalanced across pups with or without cortisol treatment. In some tests, test chambers were wiped with a $70 \%$ ethanol prior to use and before subsequent tests for removing any odors from the previous subject. All behaviors and locomotor activities of pups were recorded 
A

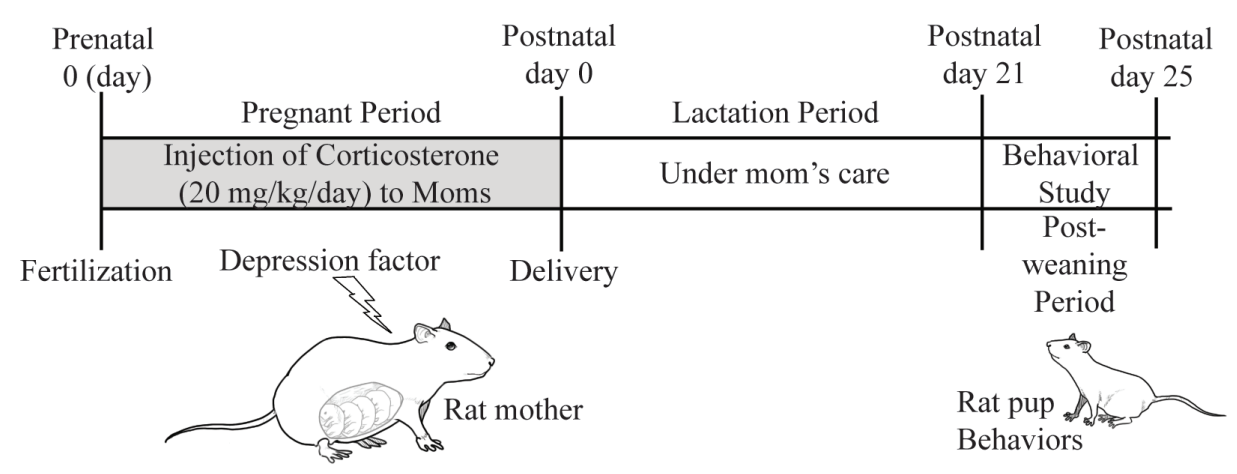

B

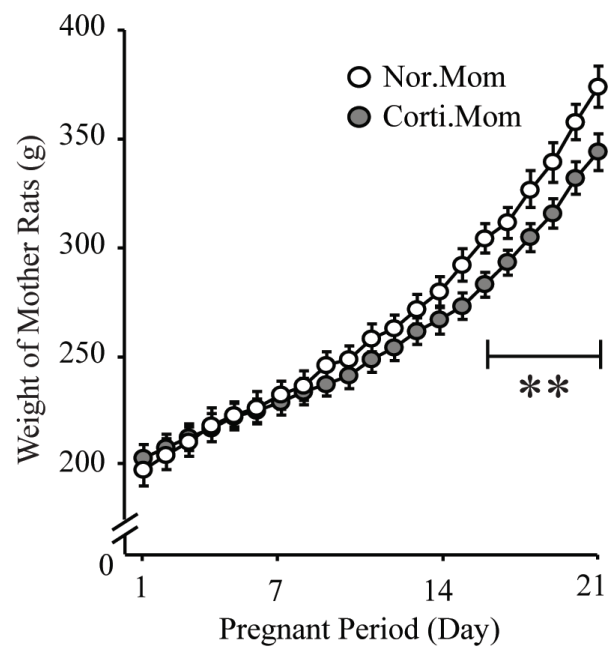

C

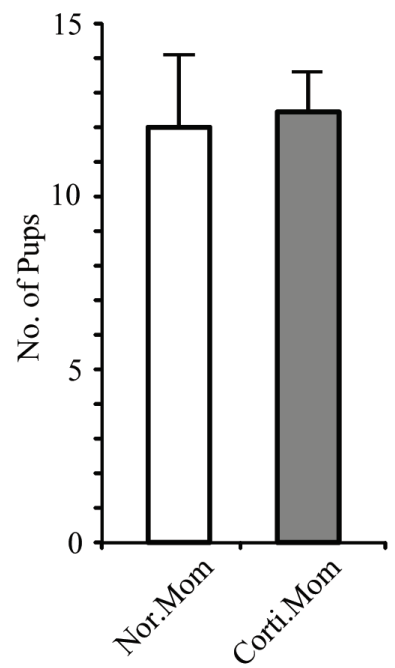

D

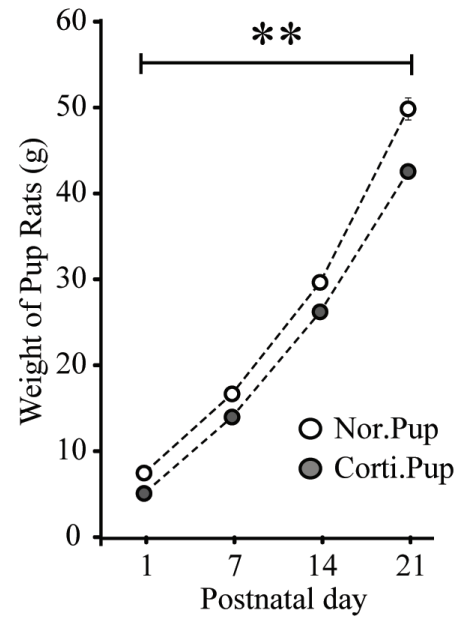

Fig. 1. The treatment of corticosterone reduces the weight of both pregnant rat mothers and postnatal pups. (A) The experimental schematic for corticosterone injection and behavioral tests. (B) Changes of body weight of rat mothers during pregnancy. On the third week, the reduced weight gain was observed in Corti.Moms. (C) The number of litters. (D) Changes in the weight gain of pup rats during lactation. The weight of Corti.Pups was significantly lower than that of Nor.Pups for three weeks. Data indicate the mean \pm S.E. ${ }^{* *} \mathrm{p}<0.01$.

using action cameras (AS30 of Sony and Yi of Xiaomi) and tracked for analysis using idTracker software (Ver. 4.97, idtracker.es/home).

\section{Forced swim (FS) test}

FS test is a well-known method used to evaluate depressive-like behaviors characterized by anxiety and helplessness [9]. FS test in this study was performed using a cylinder tank $(40 \mathrm{~cm}$ diameter $\times 60 \mathrm{~cm}$ height) that was constructed of transparent plexiglass. The tank was filled with enough water (at least $30 \mathrm{~cm}$ depth) so that animal subjects were unable to reach the bottom of the cylinder. The total height was high enough to prevent rat pups from escaping from the tank. The water temperature was set at $24.5 \pm 1^{\circ} \mathrm{C}$. The FS test was performed once a day from postnatal day 21 to day 23 and the pups swam freely for 5 minutes in each test. Behavioral patterns in the FS test were evaluated based on immobility time, swimming time and the number of climbing trials. As previously described [9], immobility, swimming and climbing were defined as absence of movement except for balancing, continuous movements of arms and legs to explore on the water surface, and movements necessary to try to climb the cylinder wall, respectively.

\section{Open field (OF) test}

The spontaneous locomotor activities were measured via $\mathrm{OF}$ test. The box used in the OF test was a cube without ceiling $(80 \times 80$ with height $50 \mathrm{~cm}$ ), which was constructed of white PVC fomex ( $5 \mathrm{~mm}$ thickness). The bottom of the open cube was divided into 16 small squares using 3 lines horizontally and vertically and four small squares located in the center were defined as the center area. 
The room temperature was set at $22 \sim 24^{\circ} \mathrm{C}$. The test was performed once a day from postnatal day 21 to day 23 and rat pups travelled freely in the box for 5 minutes in each test. Behavioral patterns in the OF test were evaluated by measuring the total travel distance, the central travel distance, the center-to-total distance ratio, and the moving velocity.

\section{Elevated plus maze (EPM) test}

EPM was employed to evaluate anxiety-like behaviors. The EPM apparatus was made of white and black PVC fomex $(5 \mathrm{~mm}$ thickness). It consisted of two sets of cross-shaped arms located on the table with the height of $70 \mathrm{~cm}$ above the ground. The width and length of each arm were $8 \mathrm{~cm}$ and $40 \mathrm{~cm}$, respectively. One set of arms fabricated from black PVC fomex, was enclosed within walls (closed arms), and the other made of white PVC fomex was exposed (open arms). The EPM test was performed once a day from postnatal day 21 to day 25 . Each pup was placed at the center of the maze and was given 5 minutes to freely explore in each test. Behavioral assessments were performed by measuring the spent time and the travel distance in open arms because rodents naturally exhibit a preference for darkness. Also, the number to reach the end of the open arm was counted and defined as extremity.

\section{Modified Morris water maze (MWM) test}

MWM is useful in assessing spatial, cognitive and memory functions based on behavioral activities in animal models. In this study, the MWM test was conducted in a metal cylinder chamber measuring $120 \mathrm{~cm}$ in diameter and $50 \mathrm{~cm}$ in depth. The tank was filled with water suspended in milk to hide the platform that was made of transparent plexiglass. The water temperature was set to $24.5 \pm 11^{\circ} \mathrm{C}$. The total water was deep enough, so that the rat was unable to touch the bottom. The hidden platform (the square shape with $100 \mathrm{~cm}^{2}$ ) was submerged $1 \sim 2 \mathrm{~cm}$ below the surface in the center of north quadrant. The rat was initially placed in the center of south quadrant and allowed to freely swim for 5 minutes in each test. MWM test was performed once a day from postnatal day 21 to day 25 to evaluate learning and memory function. Behavioral assessments were performed by measuring the escape time, the total travel distance, and the movement velocity. Furthermore, the first annulus time to reach the hidden platform, the number of annulus visits, and the spent time and travel distance in the target quadrant where the hidden platform was located, were analyzed to evaluate anxiolytic behaviors and attention deficit.

\section{Serum collection and cortisol measurement}

Serum cortisol ELISA (Rat cortisol ELISA kit, EKC31485, Biomatik co. ltd., USA) was used to measure the plasma cortisol level ac- cording to the manufacturer's protocol. Blood was collected from the jugular vein of rat mothers immediately after delivery and that of postnatal 10-day pups.

\section{Statistical analysis}

Data analysis and statistical significance were performed using Excel (Microsoft) software and SPSS. All data were presented as the mean \pm standard error of the mean (SEM), and statistical analysis was performed by one- or two-way ANOVA. The differences between the groups were considered significant when the $p$ value was $<0.05$ or $<0.01$.

\section{RESULTS}

\section{Repetitive injection of corticosterone during pregnancy reduces the weight of rat pups}

In order to increase the serum cortisol level in rat mothers and pups, corticosterone $(20 \mathrm{mg} / \mathrm{kg})$ was subcutaneously injected into the pregnant rats once a day during 21 days from confirming fertilization to delivery (Fig. 1). Saline $(0.9 \% \mathrm{NaCl})$ was injected in the control group for comparison. During pregnancy, the changes in weight gain of rats were continuously monitored and those of pups were measured after delivery (Fig. 1B and D). The difference in litter size between groups was monitored to observe the effect of cortisol on pregnancy (Fig. 1C). After birth, pups were under maternal care for 21 days and behavioral tests were performed after weaning (Fig. 1A). In this experiment, we confirmed that the weight of Corti.Moms was significantly lower than that of Nor.Moms in the late pregnant period (Fig. 1B, $\mathrm{F}=23.598, \mathrm{p}<0.01,21^{\text {st }}$ day; Nor.Mom=371.67 \pm 9.53 , $\mathrm{n}=21$; Corti. Mom=318.62 $\pm 24.62 \mathrm{~g}, \mathrm{n}=23$ ). However, the difference in weights between two mother groups was not due to the amount of fetuses (Fig. 1C, Nor.Mom=12.00 \pm 2.10 , Corti.Mom=12.44 \pm 1.16 pups per mother). Interestingly, the weights of Corti.Pups, which were measured every week after birth, were also significantly lower than that of Nor.Pups (Fig. 1D, F=65.263, $\mathrm{p}<0.01$, post-natal 21 ${ }^{\text {st }}$; Nor. Pup $=49.85 \pm 1.29, n=39$; Corti.Pup $=42.55 \pm 0.88$ g, $n=58$ ). Particularly, the significant difference in the weight of pups on post-natal day 1 indicated that the repetitive injection of corticosterone into pregnant rats influenced the fetal development.

\section{Cortisol level is increased in both Corti.Mom and Corti.Pup}

High cortisol level was critically associated with weight loss in animal models showing depressive symptoms that were induced by repetitive corticosterone injections $[9,10]$. Consistent with those reports, we also confirmed the weight loss in Corti.Moms during pregnancy and postnatal Corti.Pups, suggesting that ma- 


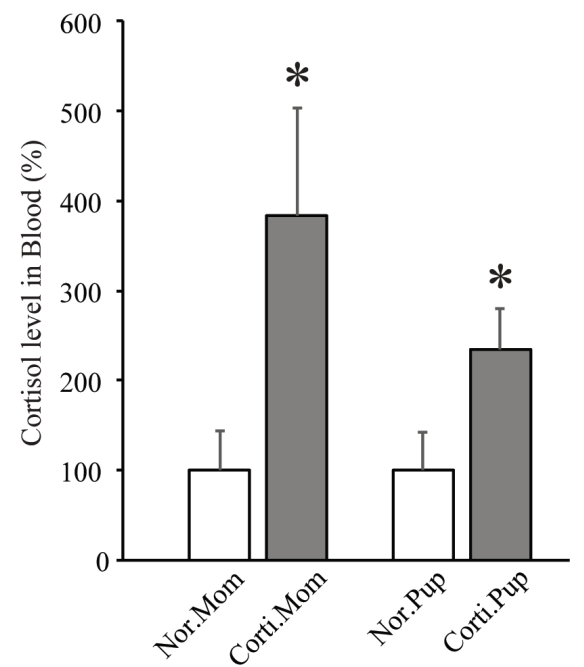

Fig. 2. The level of plasma cortisol increases in both Corti.Moms and Corti.Pups. Cortisol level was measured in blood collected from the jugular vein of rat mothers right after delivery and postnatal 10-day pups, using serum cortisol ELISA assay. Data indicate the mean \pm S.E. ${ }^{*} \mathrm{p}<0.05$.

ternal cortisol level during pregnancy might influence the cortisol level in offspring (Fig. 1). In Fig. 2, we measured the serum cortisol level in blood collected from jugular vein of rat mothers immediately after delivery and of 10 to 11-day-old pups, using ELISA. The serum cortisol levels were significantly elevated in both Corti. Moms and Corti.Pups (Fig. 2, Corti.Mom=383.31 \pm 120.47 , $p<0.05$; Corti.Pup $=234.18 \pm 45.93 \%, \mathrm{p}<0.05$; trial $\#=4$ ). This clearly demonstrated that the elevation in cortisol level of Corti.Pups was attributed to the repetitive injection of corticosterone into rat mothers during pregnancy.

\section{Corti.Pups exhibit hyperactive behavior in FS test}

The FS test is generally used to determine depressive behavior in animal models of psychiatric disorders [20,21]. Here, we used the FS test to determine behavioral abnormalities in pups because high cortisol level is critically correlated with depressive behavioral patterns in adult rodents $[9,10]$. From postnatal day 21 to day 23 , the pups were subjected to the FS test once a day, and each test was performed for 5 minutes. In results, Corti.Pups showed a significantly shorter immobility time than Nor.Pups in the second and third FS tests (Fig. 3A, F=9.234, p $<0.01,2^{\text {nd }}$ day trial; Nor. Pup $=77.61 \pm 18.62, n=10$; Corti.Pup=16.22 $\pm 7.08 \mathrm{~s}, \mathrm{n}=8$ ). In contrast, the swimming time was longer in Corti.Pups than in Nor.Pups (Fig. $3 B, F=8.731, p<0.01,2^{\text {nd }}$ day trial, Nor.Pup $=230.69 \pm 15.80$, Corti. Pup $=283.41 \pm 5.32 \mathrm{~s}$ ). The reduced immobility and higher number of swimming trials indicated possible hyperactivity of Corti. Pups compared with Nor.Pups. In this experiment, there were no statistically significant differences between Nor.Pups and Corti.
A
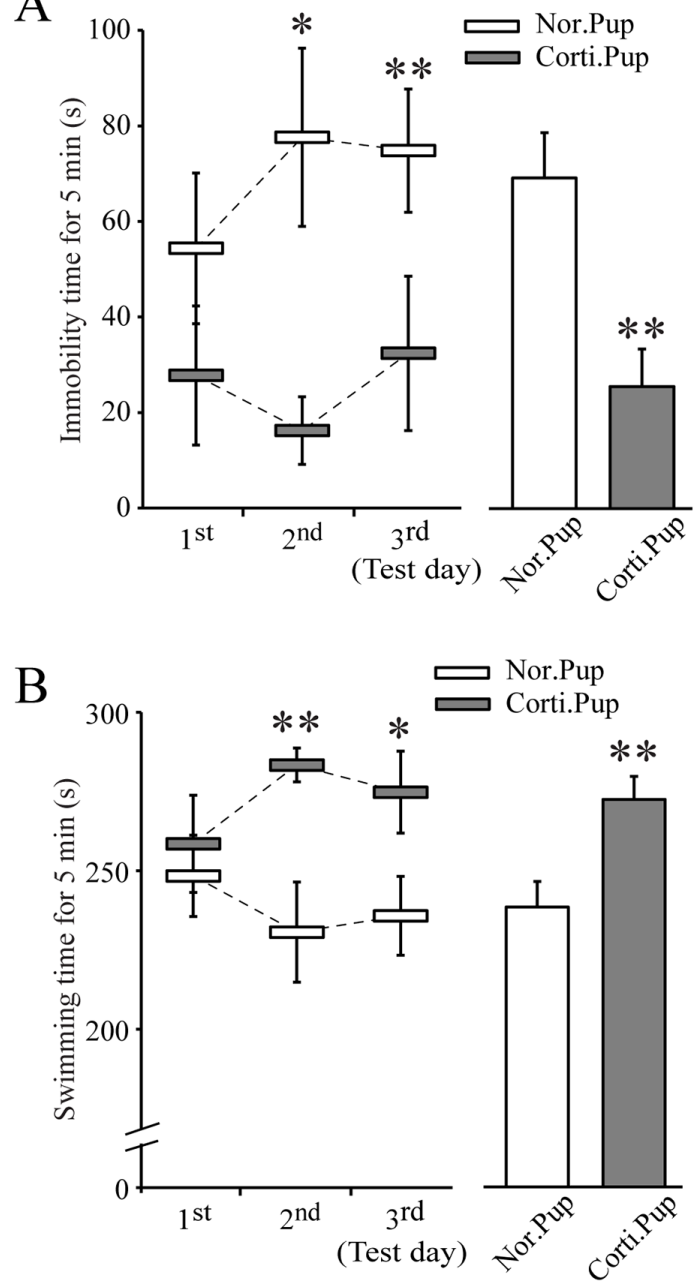

C

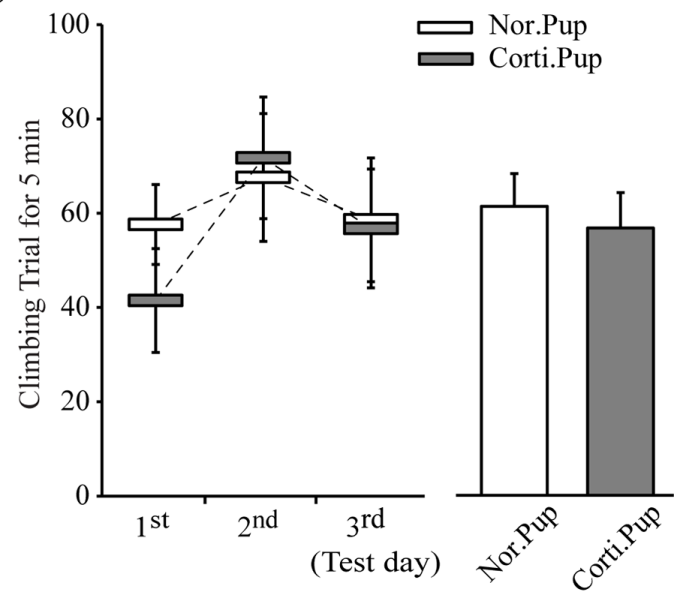

Fig. 3. Corti.Pups exhibit hyperactive behaviors in FS test. (A) The different changes of immobility time between two groups. Corti.Pups continuously moved and swam in FS test for three days of the test, so their immobility was significantly less than that of Nor.Pups. (B) The different changes of swimming time between two groups. (C) The number of climbing trials. Bar graphs in A, B and C indicate the averaged value for three test days. Data indicate the mean \pm S.E. ${ }^{*} \mathrm{p}<0.05 ;{ }^{* *} \mathrm{p}<0.01$. 
Pups in the number of climbing trials (Fig. 3C, $2^{\text {nd }}$ day trial, Nor. Pup $=67.6 \pm 13.55$, Corti.Pup $=71.75 \pm 12.91$ ). These results suggested that, unlike depressive adult models, the increased cortisol level might mediate hyperactivity in postnatal rat pups.

A

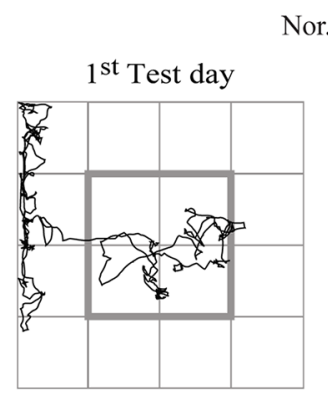

Nor.Pup

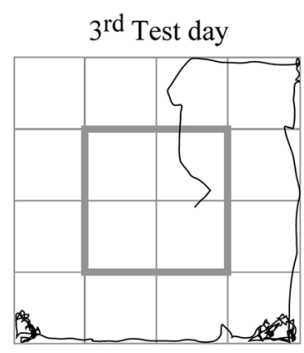

\section{Corti.Pups exhibit increased spontaneous locomotor activities in OF test}

The OF test is an useful method to experimentally determine the level of locomotor activity, anxiety, and desire to explore in
B

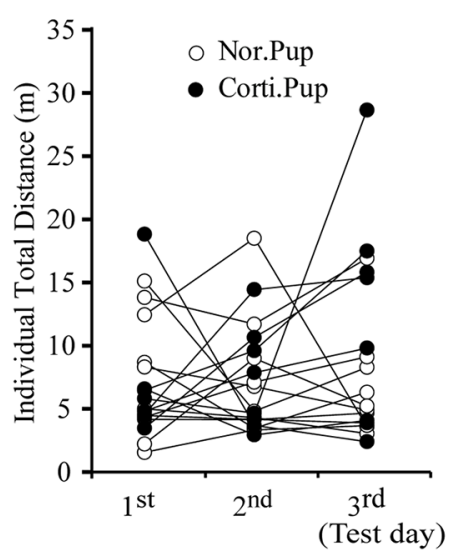

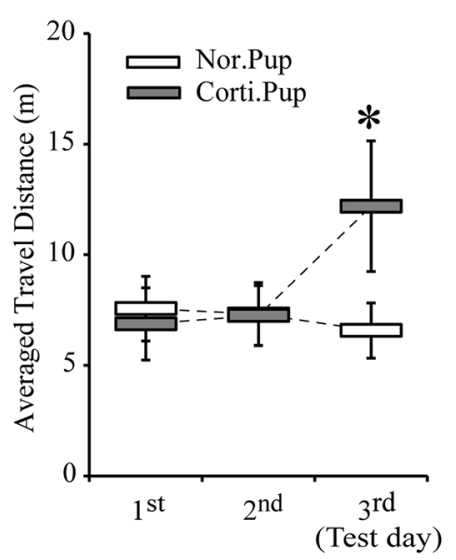

Corti.Pup

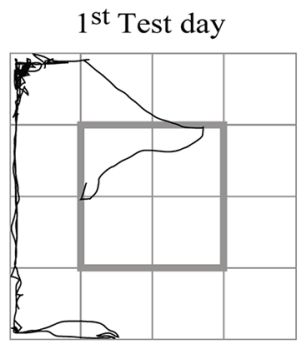

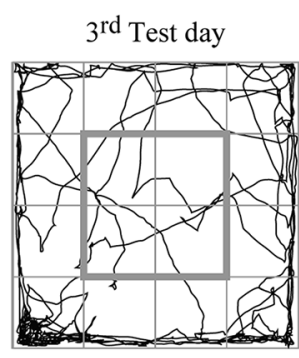

C

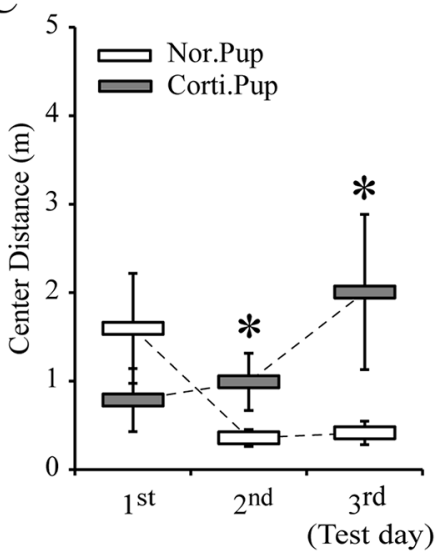

D

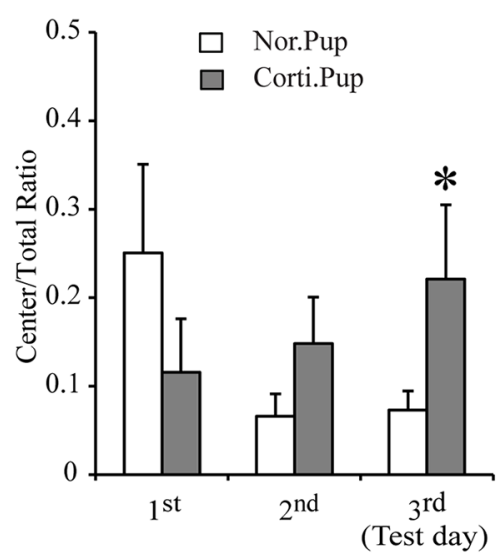

F

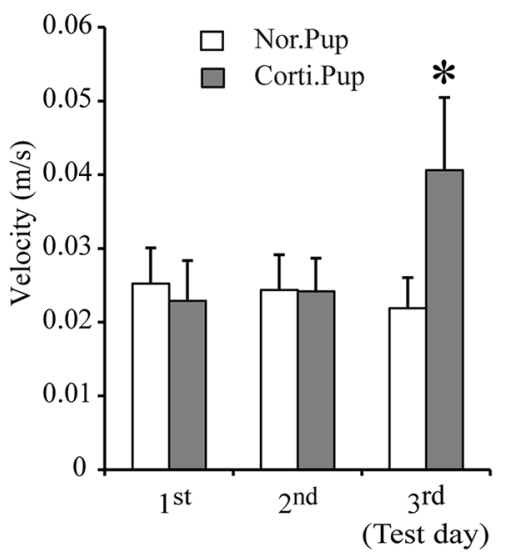

Fig. 4. Corti.Pups exhibit higher spontaneous locomotor activities in OF test. (A) Example traces of spontaneous movements in the open box. Four squares located in the center (bolded) were defined as 'center area'. Traces were tracked by idTracker software. (B) Changes of total movement distance during the test. Corti.Pups showed the increased distance on the $3^{\text {rd }}$ test day. (C) Changes of movement distance in the center area. The movement distance of Corti.Pups in the center area was significantly longer than that of Nor.Pups on the $2^{\text {nd }}$ and $3^{\text {rd }}$ test days. (D) The ratio of center distance to the total distance. The ratio of center movement in Corti.Pups was significantly increased on the $3^{\text {rd }}$ test day. (E) Changes of movement velocity in OF test. Corti.Pups exerted the increased velocity while Nor.Pups showed consistent velocity during the test. Data indicate the $m e a n \pm S$.E. ${ }^{*} \mathrm{p}<0.05$, ${ }^{* *} \mathrm{p}<0.01$. 
animal studies of psychiatric disorders [22, 23]. We also used OF tests to determine possible differences in spontaneous locomotor activities and anxiety-like behaviors of rat pups in an open environment. Rat pups were placed in an open box for 5 minutes once a day from postnatal day 21 to day 23 , and their spontaneous movements were recorded and tracked using idTracker software (Fig. 4A). In this behavioral experiment, no differences were observed between the two groups in terms of the total movement distance and central movement distance in the first test (Fig. 4B and C, Total distance, $1^{\text {st }}$ Test day, Nor.Pup $=7.56 \pm 1.47, \mathrm{n}=10$; Corti. Pup $=6.87 \pm 1.63 \mathrm{~m}, \mathrm{n}=8$ ). However, spontaneous locomotor activity was significantly increased in Corti.Pups, which exhibited longer travel distance in both total and center areas in the third test (Fig. $4 \mathrm{~B}$ and C, Total distance, $3^{\text {rd }}$ Test day, Nor.Pups $=6.57 \pm 1.24$, Corti.Pup $=12.19 \pm 2.95 \mathrm{~m}, \mathrm{~F}=6.451$, $\mathrm{p}<0.05$; Center distance, Nor. Pups $=0.41 \pm 0.13$, Corti.Pup $=2.01 \pm 0.88 \mathrm{~m}, \mathrm{~F}=8.016, \mathrm{p}<0.05$ ). Unlike Corti.Pups, Nor.Pups exhibited stable and consistent locomotor activities as well as reduced central movement for three days. These results strongly suggested that hyperactive and anxiolytic behaviors might be triggered in Corti.Pups.

\section{Corti.Pups show hyperactivity and anxiolytic behavior in EPM test}

Corti.Pups exhibited less immobility and more spontaneous movement in FS and OF tests, respectively (Fig. 3 and 4). Although representative behavioral patterns in the two tests appeared to be anxiolytic hyperactivity or impulsivity, it was still possible that anxiety-like behaviors and maladaptation were expressed as hyperactivities depending on the test environment [24]. Therefore, we further investigated if hyperactive locomotor activities of Corti.Pups were correlated with maladaptation or emotional anxiety. For this, we observed behavioral patterns in EPM which had two open arms and two closed arms at right angle (Fig. 5). This test was also performed for 5 minutes once a day from postnatal day 21 to day 25 . The spent time and travel distance in the open arms, the extremity representing the number of attempts to reach the end of an arm, and the moving velocity were measured and compared between two groups for 5 days (Fig. 5B E). The Corti.Pups exhibited longer spent time and travel distance in open arms than Nor.Pups (Fig. 5B, F=7.539, p $<0.05,5^{\text {th }}$ Test day, Spent time, Nor.Pup $=40.17 \pm 8.99, \mathrm{n}=10$; Corti.Pup $=141.05 \pm 39.20$ s, n=7; Fig. 5C, F=16.274, p $<0.01,5^{\text {th }}$ Test day, Travel distance, Nor. $P u p=216.05 \pm 24.86$, Corti.Pup $=633.37 \pm 110.58 \mathrm{~cm}$ ). These results demonstrated that Corti.Pups exhibited hyperactive behaviors, compared with Nor.Pups. Furthermore, the severe fluctuation in spent time and travel distance for 5 days of the test was observed in Corti.Pups, while Nor.Pups showed less variation. This suggested that the behaviors of Corti.Pups might reflect anxiolytic emotion and attention deficit. The extremity analysis was more evident for hyperactive and anxiolytic behaviors of Corti.Pups, because they exhibited more frequent travels to the end of the arms on days $4^{\text {th }}$ and $5^{\text {th }}$ of the test (Fig. 5D, F=24.680, p $<0.01,5^{\text {th }}$ Test day, Nor. Pup $=1.0 \pm 0.24$, Corti.Pup $=5.14 \pm 0.79$ ). The results of EPM test suggested that the behavioral phenotypes of Corti.Pups might be correlated with hyperactive behavior reflecting anxiolytic emotion. Also, the fluctuation in behavioral patterns exhibited by Corti. Pups despite repetitive trials, suggested a possible dysfunction in developing brain mediated with abnormal spatial cognition. In contrast, there were no significant differences in movement velocity $(\mathrm{cm} / \mathrm{s})$ between the two groups, indicating absence of motor function deficits (Fig. 5E, $5^{\text {th }}$ Test day, Nor.Pup $=6.50 \pm 0.67$, Corti. Pup $=5.89 \pm 0.87 \mathrm{~cm} / \mathrm{s}$ ).

\section{Corti.Pups show declines in spatial cognition and learning}

After weaning on the postnatal day 21, the modified MWM test was performed to evaluate the spatial cognitive and learning functions in rat pups. Pups were subjected to the MWM test for 5 minutes once a day from postnatal day 21 to day 25 . The representative swimming patterns of each group are shown in Fig. 6A. Escape time and total distance to reach the hidden platform and stop swimming were significantly longer in Corti.Pups than in Nor.Pups on the first test day when no difference in swimming velocity was observed (Fig. 6B D, Escape time, $\mathrm{F}=38.432$, $\mathrm{p}<0.01$, $1^{\text {st }}$ Test day, Nor.Pup $=187.81 \pm 42.20, n=7$; Corti.Pup $=508.36 \pm 48.60$ $\mathrm{s}, \mathrm{n}=11$; Total travel distance, $\mathrm{F}=22.749, \mathrm{p}<0.01,1^{\text {st }}$ Test day, Nor. Pup $=38.22 \pm 9.44$, Corti.Pup=76.56 $\pm 8.07 \mathrm{~m}$; Velocity, $\mathrm{F}=34.715$, $\mathrm{p}<0.01,1^{\text {st }}$ Test day, Nor.Pup $=0.18 \pm 0.02$, Corti.Pup $=0.15 \pm 0.01$ $\mathrm{m} / \mathrm{s}$ ). This result indicated that Corti.Pups had reduced spatial cognitive function rather than anxiolytic behavior and hyperactivity. However, in the second and third tests, Corti.Pups exhibited significantly slower swimming velocity with the escape time and total distance that were still significantly longer than those of Nor. Pups (Fig. 6B D, $3^{\text {rd }}$ Test day, Escape time, Nor.Pup=62.97 \pm 14.21 , Corti.Pup $=290.20 \pm 64.70 \mathrm{~s}, \mathrm{p}<0.05$; Total travel distance, Nor. Pup $=16.99 \pm 4.02$, Corti.Pup $=61.11 \pm 13.83 \mathrm{~m}, \mathrm{p}<0.05$; Velocity, Nor.Pup $=0.26 \pm 0.02$, Corti.Pup $=0.21 \pm 0.01 \mathrm{~m} / \mathrm{s}, \mathrm{p}<0.05$ ). This suggested that the reduced cognitive function of Corti.Pups might be correlated with anxiolytic emotion and attention deficit. However, Corti.Pups showed a gradual improvement in escape time and total distance in the fourth and final tests, similar to Nor.Pups which steadily manifested the increased cognitive function in the learning task of the MWM test. These results suggested that Corti. Pups might have delayed and incomplete cognitive function and spatial memory formation. The possible correlation of hyperactiv- 
A

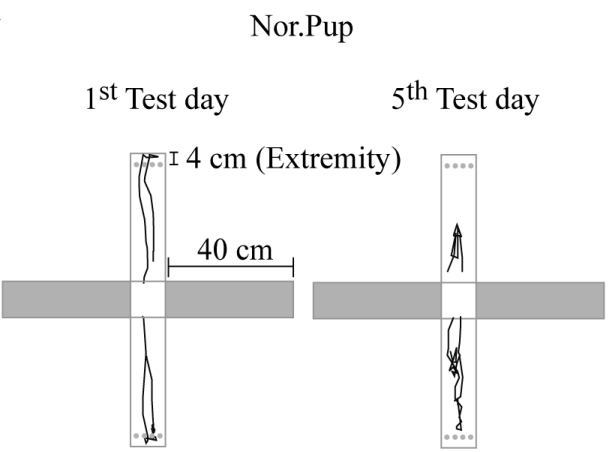

B

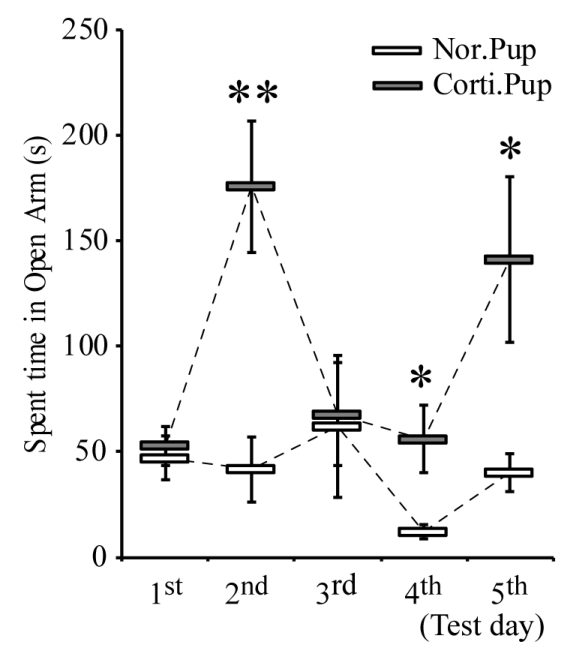

D

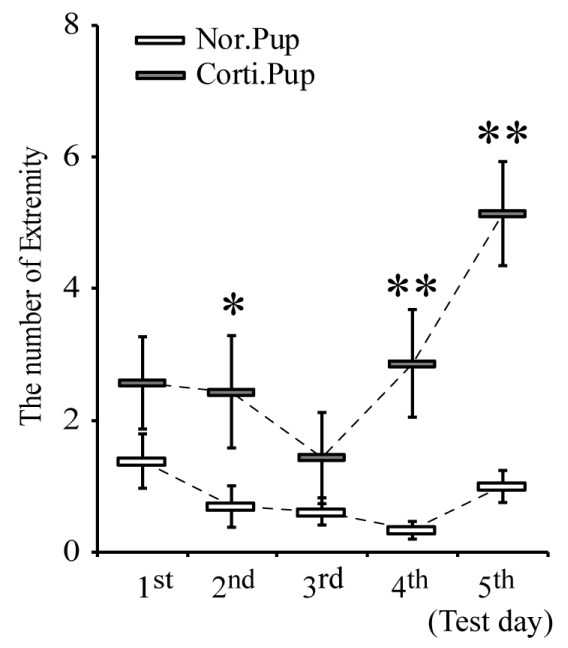

Corti.Pup

$1^{\text {st }}$ Test day $\quad 5^{\text {th }}$ Test day

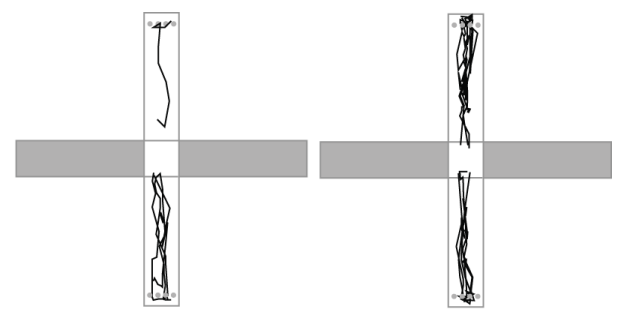

C

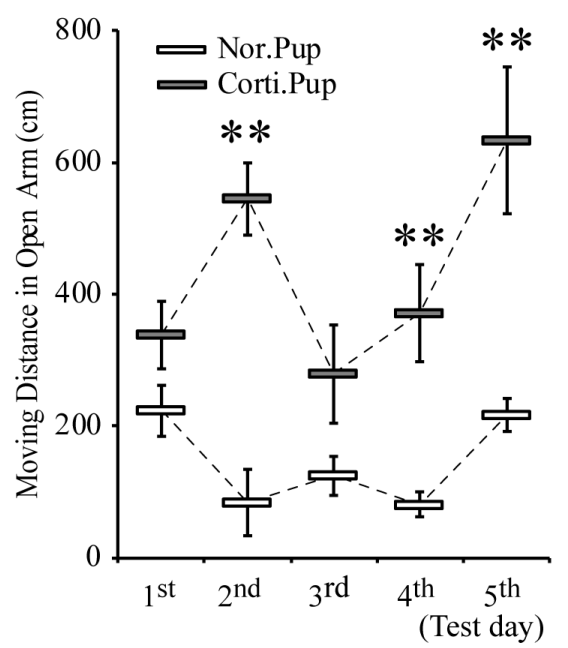

E

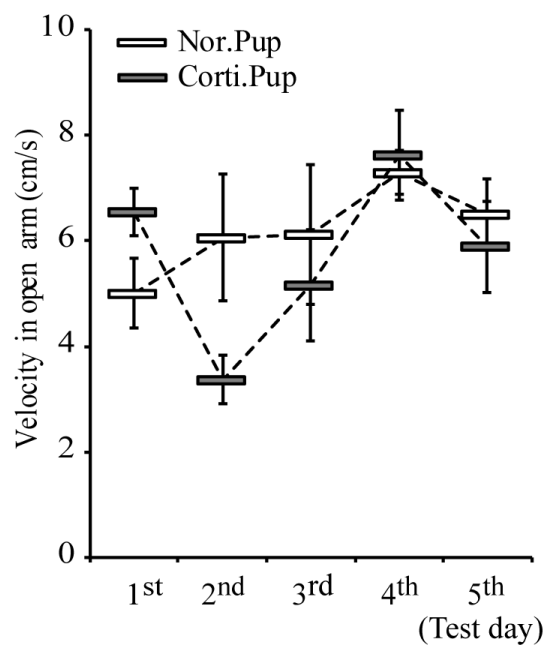

Fig. 5. Corti.Pups exert hyperactive and anxiolytic behaviors in EPM test. (A) Example traces of locomotor activities of pups in the open arms of EPM. (B, C) Changes of spent time and movement distance in the open arms during 5 days of EPM test. Corti.Pups showed longer spent time and movement distance with higher variation in the open arms, compared with Nor.Pups. (D) Changes of the extremity. During the test, attempts to touch the end of open arms was more frequently observed in Corti.Pups, demonstrating the impulsivity and anxiolytic behaviors. (E) Changes of locomotor velocity in the open arms. Data indicate the mean \pm S.E. ${ }^{*} \mathrm{p}<0.05,{ }^{* *} \mathrm{p}<0.01$. 
A
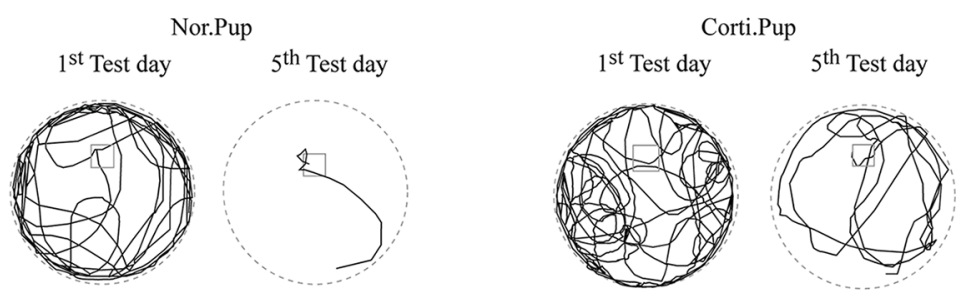

B

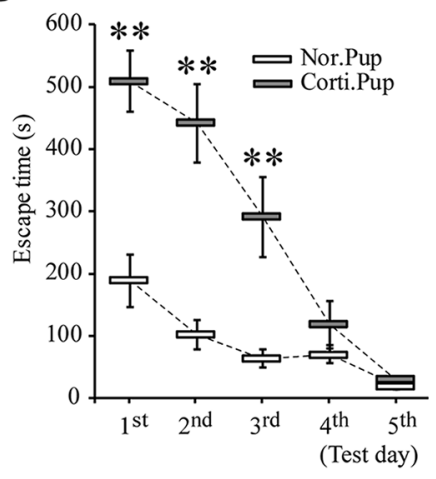

C

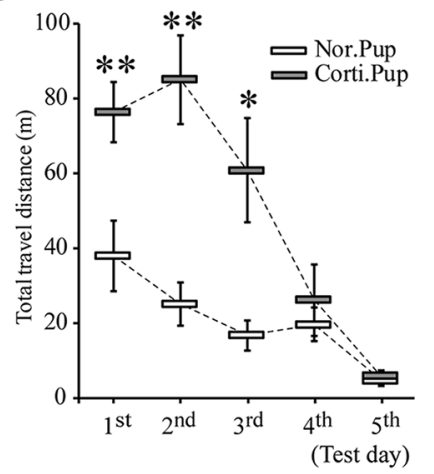

D

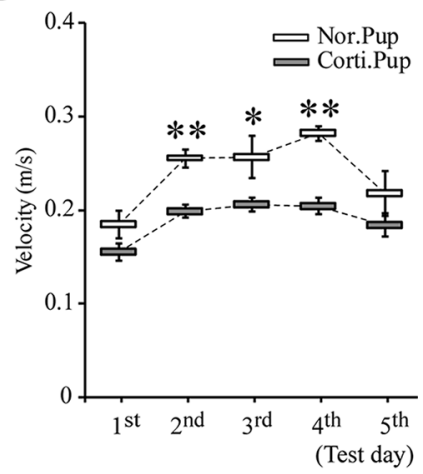

Fig. 6. Corti.Pups show hyperactive behaviors with cognitive declines in MWM test. (A) Example traces of swimming activities of pups in MWM test. $(\mathrm{B}, \mathrm{C})$ Changes of escape time and travel distance for 5 trial days of the test. Longer time and distance were observed in Corti.Pups during the initial three days, compared with Nor.Pups. However, similar with Nor.Pups, Corti.Pups rapidly found the hidden platform on the $4^{\text {th }}$ and $5^{\text {th }}$ test days, showing delayed cognitive function in the learning task. (D) Changes of swimming velocity. Data indicate the mean \pm S.E. ${ }^{*} \mathrm{p}<0.05,{ }^{* *} \mathrm{p}<0.01$.

ity and anxiolytic behavior with reduced cognitive function was supported by the analysis of annulus time and locomotor activities in the target quadrant. During 5 days of MWM test, the first annulus time to reach the hidden platform was significantly shorter in Corti.Pups than in Nor.Pups (Fig. 7A, First annulus time, $1^{\text {st }}$ Test day, Nor.Pup=149.86 \pm 31.61 , Corti.Pup=69.36 \pm 17.79 s, $p<0.01$ ). Additionally, Corti.Pups remained longer in the target quadrant area and visited the platform more frequently than Nor.Pups (Fig. 7B and C, $1^{\text {st }}$ Test day, Number of visits, Nor.Pup $=1.86 \pm 0.37$, Corti.Pup $=5.36 \pm 0.91, \mathrm{p}<0.01$; Target quadrant time, Nor. Pup $=61.14 \pm 15.30, n=8$; Corti.Pup=95.98 \pm 12.88 s, $\mathrm{p}<0.05$ ). These results indicated the possibility that Corti.Pups exhibited attention deficit during the learning task. Additionally, the delay between the first annulus visit and the final escape strongly provided evidence for impulsivity and attention deficit during learning tasks (Fig. $7 \mathrm{E}, 1^{\text {st }}$ Test day, Nor.Pup=12.32 \pm 5.56 ; Corti.Pup $=79.19 \pm 8.39 \%$, $\mathrm{p}<0.01)$.

\section{DISCUSSION}

Cortisol is the dominant factor to determine stress-mediated depression and the repetitive injection of corticosterone elevates the level of plasma cortisol, inducing neuroendocrine dysregulation and depressive symptoms in animal studies [6, 8-10, 25]. In the present study, we injected corticosterone to pregnant rats and investigated the occurrence of behavioral abnormalities in postnatal rat pups correlated with neuropsychiatric disorders such as depression or autism. Unlike adult animal models showing depressive behaviors, pups born from rats treated with corticosterone exhibited hyperactivity, anxiolytic behaviors, and impulsivity. This phenomenon was confirmed by the behavioral patterns of Corti. Pups showing 1) less immobility and increased swimming trial in FS test, 2) increased travel distance in both total and central areas of the $\mathrm{OF}$ test, 3) longer spent time and travel distance in the open arm of EPM test, 4) increased extremity in EPM test, and 5) longer escape time and swimming distance despite frequent annulus visits in MWM tests. Furthermore, Corti.Pups showed partial cognitive impairment and delayed spatial memory in repetitive MWM tests. These results provide direct evidence suggesting that cortisol-mediated dysregulation of pregnant rats may affect the behavioral and cognitive functions of their pups during postnatal development.

The high level of plasma cortisol is an important biomarker of major depressive disorders clinically as the excessive stress correlated with human depression elevates the cortisol level $[25,26]$. Therefore, it has been believed that constant exposure to external stressors may affect the neuroendocrine system of brain and induce neurochemical dysregulation resulting in neuropsychiatric 
A

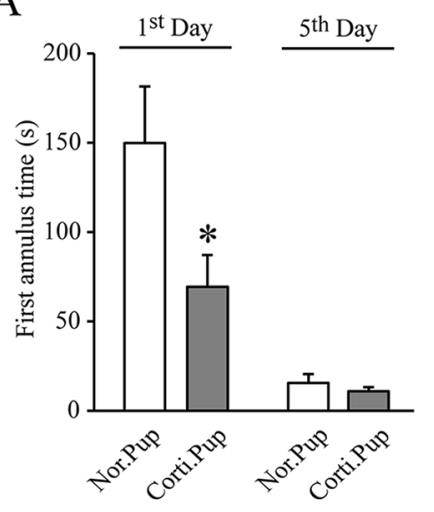

D

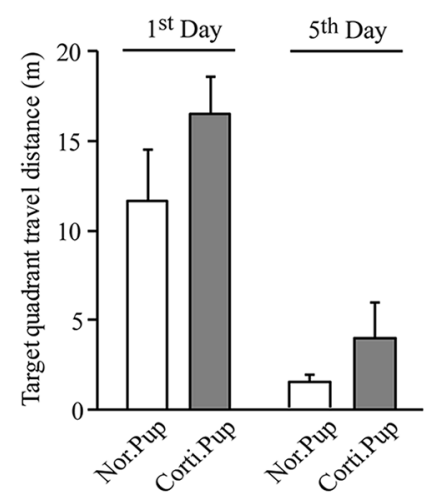

B

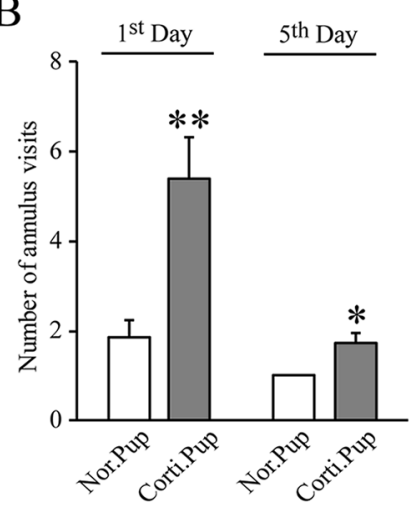

$\mathrm{E}$

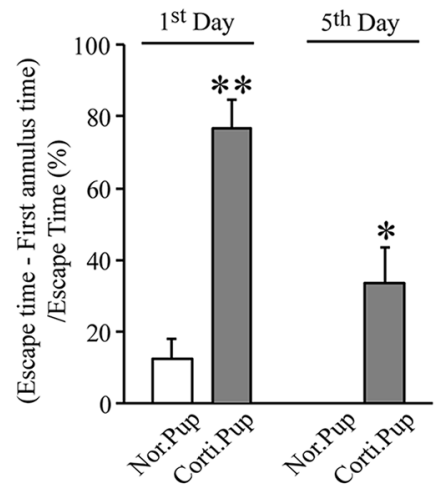

Fig. 7. Corti.Pups exert anxiolytic behaviors with attention deficit in MWM test. (A) Different annulus time between two groups. In the first test, the first annulus time to visit the hidden platform was significantly shorter in Corti.Pups than Nor.Pups. (B) The difference of annulus visit numbers. Corti. Pups more frequently visited the hidden platform than Nor.Pups. (C, D) The differences of spent time and travel distance in the target quadrant. In this analysis, Corti.Pups stayed longer in the target quadrant, demonstrating hyperactive and anxiolytic movements to explore the water maze. (E) The difference of escapes time taken since the first annulus visit. Corti.Pups showed significantly longer escape time despite the first annulus time was significantly shorter, indicating the possibility of attention deficit. Data indicate the mean \pm S.E. ${ }^{*} \mathrm{p}<0.05,{ }^{* *} \mathrm{p}<0.01$.

disorders [5, 6, 26]. In animal models, depression-like behaviors and emotions were induced by experimental manipulations to elevate the cortisol level, indicating that cortisol is a major factor triggering or exacerbating depressive symptoms $[9,10,27]$. These previous studies reported behavioral phenotypes of cortisolmediated depression in animal models, which were characterized by increased immobility, reduced locomotor activities, reduced food intake and weight gain, and increased anxiety-like emotions. These behavioral and emotional symptoms in animal models are consistent with those of patients with depressive disorders, and therefore, cortisol-induced cellular and systemic alterations in brain are key factors to explain the incidence of depression. In this study, the repetitive injection of corticosterone to pregnant rats significantly increased cortisol levels in both mothers and their pups (Fig. 2). This pharmacological manipulation exhibited the effect to acutely increase the cortisol level in adult and adolescent rat models, so most behavioral tests were performed immediately after the last injection [28-30]. However, using rat pups that were prenatally exposed to high cortisol, we executed behavioral tests during post-weaning period rather than immediately after birth. Also, the cortisol level of Corti.Pups at the postnatal day 10 was significantly lower than that of Corti.Mom measured immediately after delivery, possibly indicating the gradual decline of cortisol levels over the postpartum days. The cortisol level of Corti.Pups measured on postnatal day 15 was lower than the result of postnatal day 10 (not shown) and Gáll et al., previously observed that cortisol level measured 32 days after the last injection of corticosterone was completely restored to the normal level [31]. Therefore, cortisol level might return to normal or was insignificantly high during behavioral tests without any acute effects of high cortisol. Interestingly, Corti.Moms with high plasma cortisol did not exhibit any depressive symptoms during pregnancy and weaning period, showing behavioral activities and food intake like Nor. Moms which were treated with saline (data not shown). This indi- 
cates the possibility that the pharmacological increase of cortisol during pregnancy differently or insufficiently influences maternal neuroendocrine regulation. However, it was clear that the injection of corticosterone to mother rats negatively affected the physical development of fetuses (Fig. 1D), and it seemed that the difference in weights between mother groups was dominantly attributed to the different weights of their fetuses. In Fig 1B and D, Corti.Moms was approximately 10\% lighter than Nor. Moms when excluding the weights of fetuses at the prenatal day 21 . This difference in the weight of Corti.Mom was considerably less than that observed in adult rat models showing over 30\% reduction of the body weight by 21 -day injection of corticosterone $[27,29]$. This strongly suggests that pregnancy and related endocrine changes may degrade the effect of corticosterone treatment to affect the neuroendocrine system as well as emotional and behavioral activities, despite of the increase of cortisol level. It is well known that pregnancy itself is a dominant factor to affect cortisol regulation in animals and human $[32,33]$.

Pups born from Corti.Moms exhibited different behavioral patterns compared with depressive adult models. As a result, Corti.Pups exhibited consistently hyperactive movements in all behavioral tests in this study. Unlike cortisol-induced depressive behaviors, Corti.Pups showed less immobility in FS test, more spontaneous locomotor activities in OF test, longer spent time and increased distance in the open arm of the EPM test, and longer swimming time and distance in the MWM test (Fig. 3 6). These behavioral phenotypes are clearly consistent with hyperactivity in ADHD models $[19,23]$. It is also possible that increased spent time and distance in the center of the OF test and the open arms of the EPM test reflect anxiolytic and impulsive emotion of Corti. Pups (Fig. 4 and 5). In horizontal exploration tasks of animal models, the rate of exposure in open environment is a useful indicator to determine anxiety-like behaviors and emotions [22, 23, 34]. Depressive animals with anxiety tend to prefer a closed environment while increased spontaneous locomotor activities in open area are often attributed to anxiolytic emotions [35]. The decrease of anxiety-like behaviors is one of the representative phenotypes observed in ADHD models which express hyperactivity [23, 35]. Particularly, the number of trials to touch the end of the EPM open arms was significantly increased in Corti.Pups, indicating a decrease in their anxiety-like emotion (\# of extremity in Fig. 5). The impulsivity was also observed in behaviors of Corti.Pups. In EPM test, the spent time and distance in open arms varied greatly in Corti.Pups while steady and consistent locomotor activities were observed in Nor.Pups during five days of the test (Fig. 5). Thus, Corti.Pups in this study manifested behavioral phenotypes consistent with ADHD models characterized by hyperactivity and anxiolytic impulsivity [36].

In previous clinical studies, $50 \%$ of adults and $30 \%$ of children with ADHD showing the comorbidity suffered from depressive episodes and anxiety [37-39]. This clearly indicates that ADHD increase the onset of depressive symptoms in human. However, unlike human studies, it seems difficult to reliably evaluate emotional anxiety and depressive symptoms in ADHD rodent models. In this study, we observed continuous swimming in FS test, longer exposure to open conditions and increased extremity in OF and EPM tests. These behavioral patterns have been demonstrated to be mediated to hyperactive and anxiolytic emotion in many previous reports that studied about ADHD rodent models [23, 29, 30]. However, it is still necessary to identify the comorbidities in animal models with psychiatric disorders for clarifying the pathogenic mechanism underlying psychiatric disorders. Although we did not focus on the comorbidities in ADHD rodent models, our results provide evidence for a possible correlation between ADHD and depressive symptoms because the treatment of corticosterone is commonly used to induce depressive symptoms in rodents.

Although it is still debated, many previous studies reported the co-existence of cognitive impairment with ADHD symptoms in human and animal models. Learning disorders and memory deficits were observed in ADHD patients showing behavioral and social problems [40, 41]. Genetically modified rodent models exhibited cognitive dysfunction in learning tasks and lower memory retention $[23,34,35]$. SHR models of ADHD also revealed memory impairment correlated with hippocampal dysfunction [42-44], while intact spatial learning was reported in another study [45]. Here we observed defective cognitive behavior and delayed learning function in Corti.Pups, which exhibited longer escape time and swimming distance despite more frequent annulus visits in MWM test (Fig. 7). Additionally, their cognitive dysfunction appeared to be attributed to anxiolytic behaviors and attention deficit evidenced by more frequent annulus visits as well as longer travel distance in the target quadrant. Consistent with previous studies, our results strongly suggest declines of behavioral and cognitive functions in the developing brain of Corti.Pups following maternal dysregulation of neuroendocrine system that is mediated with cortisol.

Various environmental challenges such as malnutrition and stress during the prenatal period, increase susceptibility to psychiatric disorders associated with cognitive dysfunction during later life and dysregulation in development of fetal brain seriously affects the neuroendocrine system, resulting in mental and cognitive disorders $[15,46,47]$. Therefore, it is necessary to focus on abnormal processing or dysregulation in developmental brains to identify the pathogenic mechanism of ADHD which 
is a heterogeneous developmental brain disorder. Although the cortisol-mediated relationship between mother and fetus should be addressed in a further study, we here clearly demonstrated that the experimental manipulation to increase maternal cortisol level during pregnancy resulted in ADHD-like symptoms in postnatal rat pups. Our findings suggest that, in terms of brain development, Corti.Pups may be a potent experimental model of ADHD rather than other genetically modified animals.

\section{ACKNOWLEDGEMENTS}

The work was supported by the National Research Foundation of Korea (NRF) grant funded by the Korea government (MEST) (No. NRF-2016R1D1A1B0101086).

\section{REFERENCES}

1. Swales DA, Stout-Oswald SA, Glynn LM, Sandman C, Wing DA, Davis EP (2018) Exposure to traumatic events in childhood predicts cortisol production among high risk pregnant women. Biol Psychol 139:186-192.

2. Slopen N, Zhang J, Urlacher SS, De Silva G, Mittal M (2018) Maternal experiences of intimate partner violence and Creactive protein levels in young children in Tanzania. SSM Popul Health 6:107-115.

3. Keresztes A, Raffington L, Bender AR, Bögl K, Heim C, Shing YL (2020) Hair cortisol concentrations are associated with hippocampal subregional volumes in children. Sci Rep 10:4865.

4. Mateos RM, Jiménez G, Álvarez-Gil C, Visiedo F, Rivera-Rodríguez F, Santos-Rosendo C, Rodriguez-Pareja A, Perdomo G, Lechuga-Sancho AM (2018) Excess hydrocortisone hampers placental nutrient uptake disrupting cellular metabolism. Biomed Res Int 2018:5106174.

5. Nazzari S, Fearon P, Rice F, Dottori N, Ciceri F, Molteni M, Frigerio A (2019) Beyond the HPA-axis: exploring maternal prenatal influences on birth outcomes and stress reactivity. Psychoneuroendocrinology 101:253-262.

6. Fatima M, Srivastav S, Mondal AC (2017) Prenatal stress and depression associated neuronal development in neonates. Int J Dev Neurosci 60:1-7.

7. Cox EQ, Stuebe A, Pearson B, Grewen K, Rubinow D, Meltzer-Brody S (2015) Oxytocin and HPA stress axis reactivity in postpartum women. Psychoneuroendocrinology 55:164-172.

8. Braithwaite EC, Murphy SE, Ramchandani PG (2016) Effects of prenatal depressive symptoms on maternal and infant cortisol reactivity. Arch Womens Ment Health 19:581-590.
9. Iijima M, Ito A, Kurosu S, Chaki S (2010) Pharmacological characterization of repeated corticosterone injection-induced depression model in rats. Brain Res 1359:75-80.

10. Bairos-Novak KR, Ryan CP, Freeman AR, Anderson WG, Hare JF; Handling editor: Sean Lema (2018) Like mother, like daughter: heritability of female Richardson's ground squirrel Urocitellus richardsonii cortisol stress responses. Curr Zool 64:153-163.

11. Gong Y, Sun XL, Wu FF, Su CJ, Ding JH, Hu G (2012) Female early adult depression results in detrimental impacts on the behavioral performance and brain development in offspring. CNS Neurosci Ther 18:461-470.

12. Lin YL, Wang S (2014) Prenatal lipopolysaccharide exposure increases depression-like behaviors and reduces hippocampal neurogenesis in adult rats. Behav Brain Res 259:24-34.

13. Soualeh N, Dridi I, Eppe G, Némos C, Soulimani R, Bouayed J (2017) Perinatal programming of depressive-like behavior by inflammation in adult offspring mice whose mothers were fed polluted eels: gender selective effects. Brain Behav Immun 63:137-147.

14. Takai Y, Kawai M, Ogo T, Ichinose T, Furuya S, Takaki N, Tone Y, Udo H, Furuse M, Yasuo S (2018) Early-life photoperiod influences depression-like behavior, prepulse inhibition of the acoustic startle response, and hippocampal astrogenesis in mice. Neuroscience 374:133-143.

15. Marques AH, Bjørke-Monsen AL, Teixeira AL, Silverman MN (2015) Maternal stress, nutrition and physical activity: impact on immune function, CNS development and psychopathology. Brain Res 1617:28-46.

16. Salari AA, Fatehi-Gharehlar L, Motayagheni N, Homberg JR (2016) Fluoxetine normalizes the effects of prenatal maternal stress on depression- and anxiety-like behaviors in mouse dams and male offspring. Behav Brain Res 311:354-367.

17. Bogi E, Belovicová K, Ujhazy E, Mach M, Koprdova R, Zilava L, Garafová A, Jezova D, Dubovicky M (2018) Perinatal exposure to venlafaxine leads to lower anxiety and depression-like behavior in the adult rat offspring. Behav Pharmacol 29:445452.

18. Lautarescu A, Craig MC, Glover V (2020) Prenatal stress: effects on fetal and child brain development. Int Rev Neurobiol 150:17-40.

19. Kozłowska A, Wojtacha P, Równiak M, Kolenkiewicz M, Tsai ML (2019) Differences in serum steroid hormones concentrations in spontaneously hypertensive rats (SHR) - an animal model of attention-deficit/hyperactivity disorder (ADHD). Physiol Res 68:25-36.

20. Bagosi Z, Csabafi K, Balangó B, Pintér D, Szolomájer-Csikós O, 
Bozsó Z, Tóth G, Telegdy G, Szabó G (2018) Anxiolytic- and antidepressant-like actions of Urocortin 2 and its fragments in mice. Brain Res 1680:62-68.

21. Abdul Aziz NU, Chiroma SM, Mohd Moklas MA, Adenan MI, Ismail A, Hidayat Baharuldin MT (2020) Antidepressantlike properties of fish oil on postpartum depression-like rats model: involvement of serotonergic system. Brain Sci 10:733.

22. Eells JB, Lipska BK, Yeung SK, Misler JA, Nikodem VM (2002) Nurr1-null heterozygous mice have reduced mesolimbic and mesocortical dopamine levels and increased stress-induced locomotor activity. Behav Brain Res 136:267-275.

23. Montarolo F, Martire S, Perga S, Spadaro M, Brescia I, Allegra S, De Francia S, Bertolotto A (2019) NURR1 deficiency is associated to ADHD-like phenotypes in mice. Transl Psychiatry 9:207.

24. Tassan Mazzocco M, Guarnieri FC, Monzani E, Benfenati F, Valtorta F, Comai S (2021) Dysfunction of the serotonergic system in the brain of synapsin triple knockout mice is associated with behavioral abnormalities resembling synapsinrelated human pathologies. Prog Neuropsychopharmacol Biol Psychiatry 105:110135.

25. Alenko A, Markos Y, Fikru C, Tadesse E, Gedefaw L (2020) Association of serum cortisol level with severity of depression and improvement in newly diagnosed patients with major depressive disorder in Jimma medical center, Southwest Ethiopia. PLoS One 15:e240668.

26. Doolin K, Farrell C, Tozzi L, Harkin A, Frodl T, O'Keane V (2017) Diurnal hypothalamic-pituitary-adrenal axis measures and inflammatory marker correlates in major depressive disorder. Int J Mol Sci 18:2226.

27. Dwivedi Y, Roy B, Lugli G, Rizavi H, Zhang H, Smalheiser NR (2015) Chronic corticosterone-mediated dysregulation of microRNA network in prefrontal cortex of rats: relevance to depression pathophysiology. Transl Psychiatry 5:e682.

28. Li J, Xie X, Li Y, Liu X, Liao X, Su YA, Si T (2017) Differential behavioral and neurobiological effects of chronic corticosterone treatment in adolescent and adult rats. Front $\mathrm{Mol} \mathrm{Neu-}$ rosci 10:25.

29. Lee B, Sur B, Shim I, Lee H, Hahm DH (2015) Angelica gigas ameliorate depression-like symptoms in rats following chronic corticosterone injection. BMC Complement Altern Med 15:210.

30. Kong H, Zeng XN, Fan Y, Yuan ST, Ge S, Xie WP, Wang H, Hu G (2014) Aquaporin-4 knockout exacerbates corticosteroneinduced depression by inhibiting astrocyte function and hippocampal neurogenesis. CNS Neurosci Ther 20:391-402.

31. Gáll Z, Farkas S, Albert Á, Ferencz E, Vancea S, Urkon M, Kol- csár M (2020) Effects of chronic cannabidiol treatment in the rat chronic unpredictable mild stress model of depression. Biomolecules 10:801.

32. Fan JM, Chen XQ, Du JZ (2014) Prenatal stress, anxiety and depression: a mechanism involving CRH peptide family. Neuro Endocrinol Lett 35:429-439.

33. Marceau K, Wang W, Robertson O, Shirtcliff EA (2020) A systematic review of hair cortisol during pregnancy: reference ranges and methodological considerations. Psychoneuroendocrinology 122:104904.

34. Rojas P, Joodmardi E, Hong Y, Perlmann T, Ogren SO (2007) Adult mice with reduced Nurrl expression: an animal model for schizophrenia. Mol Psychiatry 12:756-766.

35. Huang D, Liu H, Zhu A, Zhou Y, Li Y (2020) Forebrain excitatory neuron-specific SENP2 knockout mouse displays hyperactivity, impaired learning and memory, and anxiolyticlike behavior. Mol Brain 13:59.

36. Sagvolden T (2000) Behavioral validation of the spontaneously hypertensive rat (SHR) as an animal model of attentiondeficit/hyperactivity disorder (AD/HD). Neurosci Biobehav Rev 24:31-39.

37. Fischer M, Barkley RA, Smallish L, Fletcher K (2002) Young adult follow-up of hyperactive children: self-reported psychiatric disorders, comorbidity, and the role of childhood conduct problems and teen CD. J Abnorm Child Psychol 30:463475.

38. Biederman J, Monuteaux MC, Mick E, Spencer T, Wilens TE, Silva JM, Snyder LE, Faraone SV (2006) Young adult outcome of attention deficit hyperactivity disorder: a controlled 10year follow-up study. Psychol Med 36:167-179.

39. Sobanski E, Brüggemann D, Alm B, Kern S, Deschner M, Schubert T, Philipsen A, Rietschel M (2007) Psychiatric comorbidity and functional impairment in a clinically referred sample of adults with attention-deficit/hyperactivity disorder (ADHD). Eur Arch Psychiatry Clin Neurosci 257:371-377.

40. Pfiffner LJ, Haack LM (2014) Behavior management for school-aged children with ADHD. Child Adolesc Psychiatr Clin N Am 23:731-746.

41. Tibu F, Sheridan MA, McLaughlin KA, Nelson CA, Fox NA, Zeanah CH (2016) Disruptions of working memory and inhibition mediate the association between exposure to institutionalization and symptoms of attention deficit hyperactivity disorder. Psychol Med 46:529-541.

42. Grünblatt E, Bartl J, Iuhos DI, Knezovic A, Trkulja V, Riederer P, Walitza S, Salkovic-Petrisic M (2015) Characterization of cognitive deficits in spontaneously hypertensive rats, accompanied by brain insulin receptor dysfunction. J Mol Psychia- 
$\operatorname{try} 3: 6$.

43. Johnson AC, Miller JE, Cipolla MJ (2020) Memory impairment in spontaneously hypertensive rats is associated with hippocampal hypoperfusion and hippocampal vascular dysfunction. J Cereb Blood Flow Metab 40:845-859.

44. Raz L, Yang Y, Thompson J, Hobson S, Pesko J, Mobashery S, Chang M, Rosenberg G (2018) MMP-9 inhibitors impair learning in spontaneously hypertensive rats. PLoS One 13:e0208357.

45. Jichao S, Xinmin H, Xianguo R, Dongqi Y, Rongyi Z, Shuang L, Yue Y, Yuchen S, Jingnan Y (2017) Saikosaponin A alleviates symptoms of attention deficit hyperactivity disorder through downregulation of DAT and enhancing BDNF expression in spontaneous hypertensive rats. Evid Based Complement Alternat Med 2017:2695903.

46. Nestler EJ, Hyman SE (2010) Animal models of neuropsychiatric disorders. Nat Neurosci 13:1161-1169.

47. Sandman CA, Davis EP, Buss C, Glynn LM (2012) Exposure to prenatal psychobiological stress exerts programming influences on the mother and her fetus. Neuroendocrinology 95:7-21 